\title{
New hydrogen atom model and radiation absorption mechanism
}

\section{Xiao En Wang ( $\nabla$ wang-xiaoen@qq.com )}

Weifang Engineering Vocational College “ (Original name: Weifang Educational College)

\section{Research Article}

Keywords: Bohr radius, new hydrogen atom model, radiation absorption mechanism, physical meaning of Planck constant, half frequency problem

Posted Date: July 6th, 2021

DOl: https://doi.org/10.21203/rs.3.rs-584713/v2

License: (c) (i) This work is licensed under a Creative Commons Attribution 4.0 International License. Read Full License 


\title{
New hydrogen atom model and radiation absorption mechanism
}

\author{
Xiao-En Wang (王孝恩) ${ }^{1}$ \\ ${ }^{1}$ Author institution: Weifang Engineering Vocational College (Weifang Educational College), \\ Qingzhou City, Shandong Weifang, China.262500. Correspondence:wang-xiaoen@qq.com.
} Abstract

Only Coulomb force is considered in Bohr model. It is found that electron in ground state hydrogen atom is also affected by Lorentz force of the same magnitude. Under the combined action of Coulomb force and Lorentz force, the radius of hydrogen atom is twice that of Bohr, and the rotation frequency of electron is half that of Bohr. The "half frequency" problem of Bohr's theory is solved, and the quantization result of the energy level of hydrogen atom is obtained by using the standing wave condition of quantum mechanics. The mechanism and details of photon absorption by hydrogen atom are analyzed and explained, and the physical meaning of Planck constant is explained.

\section{Keywords}

Bohr radius, new hydrogen atom model, radiation absorption mechanism, physical meaning of Planck constant, half frequency problem 


\section{Introduction}

As early as the $1850 \mathrm{~s}$, people found four lines of hydrogen in the visible spectrum of sunlight: $\mathrm{H}_{\alpha}$, $\mathrm{H}_{\beta}, \mathrm{H}_{\gamma}$ and $\mathrm{H}_{\delta}$. In 1884, Balmer, a middle school teacher in Switzerland, proposed a simple formula for calculating the wavelengths of these spectral lines ${ }^{[1]}$

$$
\lambda=B_{a} \frac{n^{2}}{n^{2}-4}
$$

where $n$ is any positive integer greater than 2 , and $B_{a}=3645.6 \times 10^{-10} \mathrm{~m}$.

In 1890, Johannes Robert Rydberg of Sweden independently put forward a more general formula for the spectrum of hydrogen atom ${ }^{[2]}$

$$
\widetilde{v}=\mathrm{R}_{\mathrm{y}}\left(\frac{1}{n_{1}^{2}}-\frac{1}{n_{2}^{2}}\right)
$$

where $\tilde{v}$ is the wave number of the spectral line $\left(\mathrm{cm}^{-1}\right), n_{1}$ and $n_{2}$ are positive integers, and $n_{1}<n_{2} ; \mathrm{R}_{\mathrm{y}}$ is the Rydberg constant, and the determined value in 1987 is $\mathrm{R}_{\mathrm{y}}=109737.31573(3) \mathrm{cm}^{-1}{ }^{[1]}$. If $n_{1}=2$, and let $n_{2}=n$, the formula (2) can be simplified as Balmer formula (1); If $n_{1}=1$, and let $n_{2}=n$, we obtain the Lyman series formula.

\section{Rydberg frequency and Rydberg radius for hydrogen atom}

With the speed of light $c$, let $c \tilde{v}=v$ and $c \mathrm{R}_{\mathrm{y}}=f_{\mathrm{R}}$, formula (2) can be rewritten as

$$
v=f_{\mathrm{R}}\left(\frac{1}{n_{1}^{2}}-\frac{1}{n_{2}^{2}}\right) \square \text { or } v=\frac{f_{\mathrm{R}}}{n_{1}^{2}}-\frac{f_{\mathrm{R}}}{n_{2}^{2}}
$$

Look at both sides of equation (3). The left, the $v$ is the frequency of the radiation photon. The right, the $f_{\mathrm{R}}$ should be a fundamental frequency of the atomic vibration, and here, we call it the Rydberg frequency.

$$
f_{\mathrm{R}}=c R_{\mathrm{y}}=2.998 \times 10^{8} \mathrm{~m} / \mathrm{s} \times 1.0974 \times 10^{7} \mathrm{~m}^{-1}=3.290 \times 10^{15} \mathrm{~s}^{-1} .
$$

According to modern knowledge, the electron in the ground state hydrogen atom moves in a circular orbit, and the ratio of its speed to the speed of light is a constant, which is the fine structure constant $\alpha^{[3]}$ 。

$$
v=c \alpha=2.998 \times 10^{8} \mathrm{~m} / \mathrm{s} \times 1 / 137.0=2.188 \times 10^{6} \mathrm{~m} / \mathrm{s}
$$

If $f_{\mathrm{R}}$ is a fundamental frequency, a radius corresponding the ground state orbit of hydrogen atom, $r_{\mathrm{R}}$, can be calculated 


$$
r_{\mathrm{R}}=\frac{v}{2 \pi f_{\mathrm{R}}}=\frac{2.188 \times 10^{6}}{2 \pi \times 3.290 \times 10^{15}}=1.058 \times 10^{-10}(\mathrm{~m})
$$

Here, we call $r_{\mathrm{R}}$ the Rydberg radius. In formula (2), $\frac{f_{\mathrm{R}}}{n^{2}}$ is a frequency corresponding the orbital resonance of hydrogen atom at the $n$th level.

The $r_{\mathrm{R}}$ value obtained here is obviously different from the orbital radius of the ground state hydrogen atom in the current textbooks. In fact, it is only inconsistent with the Bohr radius in Bohr model named after a Danish physicist, Niels Bohr. This issue will continue to be discussed in section 3 , and resolved in section 4. In this paper the solution for this problem will be a great breakthrough for the current theory, and also become the theoretical basis for discussing the mechanism of atomic absorption radiation.

\section{Bohr model and Bohr radius}

In 1913 Bohr assumed that electrons in atoms move in some stable orbits, different orbits belong to different energy levels, and that electrons moving in these stable orbits neither absorb nor radiate photons; Electrons absorb or radiate photons only when they jump between different levels. He first dealt with the hydrogen atom, and the lowest energy orbit is called the ground state ${ }^{[4]}$.

When an object moves in a circle with velocity $(v)$, if the radius is $r$, the centripetal force $\left(F_{\mathrm{X}}\right)$ it receives is

$$
F_{\mathrm{X}}=\frac{m v^{2}}{r}
$$

where $m$ is the electron mass when it is in hydrogen atom. According to Newton's third law, Bohr thinks that the Coulomb force $F_{\mathrm{C}}$ between electron and proton provides centripetal force to balance the circular motion.

$$
F_{\mathrm{C}}=\frac{e^{2}}{4 \pi \varepsilon_{0} r^{2}}
$$

where $e$ is the charge of proton or electron, $\varepsilon_{0}$ is the dielectric constant in vacuum. At that time, Bohr thought that in an equilibrium system of hydrogen atom, $F_{\mathrm{C}}=F_{\mathrm{X}}$, and by combining formula (7) and formula (8), the following formula is got 


$$
\frac{e^{2}}{4 \pi \varepsilon_{0} r_{\mathrm{B}}}=m v^{2}
$$

For the hydrogen atom in the ground state, all the physical quantities in formula (9) are constants. According to either the quantization condition supposed by Bohr or formula (5), $r_{\mathrm{B}}$ can be calculated.

$$
\begin{aligned}
& r_{\mathrm{B}}=\frac{e^{2}}{4 \pi \varepsilon_{0} m v^{2}} \\
& =\frac{\left(1.602 \times 10^{-19}\right)^{2}}{4 \pi \times 8.854 \times 10^{-12} \times 9.110 \times 10^{-31} \times\left(2.188 \times 10^{6}\right)^{2}}=5.29 \times 10^{-11}(\mathrm{~m})
\end{aligned}
$$

The $r_{\mathrm{B}}$ value is only a little larger than the covalent radius of hydrogen, so the calculated value of Bohr has been considered reasonable. Bohr also derived the expression of Rydberg constant by known physical constants ${ }^{[4]}$

$$
R_{y}=\frac{2 \pi^{2} m e^{4}}{\left(4 \pi \varepsilon_{0}\right)^{2} h^{3} c} \square \text { or } R_{y}=\frac{m e^{4}}{8 \varepsilon_{0}^{2} h^{3} c}
$$

Obviously, Bohr's success began with "general mechanics". However, he also encountered a lifelong trouble. The main problem is that $r_{\mathrm{B}}$ is only half of the Rydberg radius $r_{\mathrm{R}}$ calculated in (6) above. In other words, Bohr's calculation of the circular orbital frequency $\left(f_{\mathrm{B}}\right)$ for the ground state of hydrogen atom is twice as large as the above Rydberg frequency $\left(f_{\mathrm{R}}\right)$ by formula (4).

$$
f_{\mathrm{B}}=\frac{v}{2 \pi r_{\mathrm{B}}}=\frac{2.188 \times 10^{6}}{2 \pi \times 5.29 \times 10^{-11}}=6.583 \times 10^{15}\left(\mathrm{~s}^{-1}\right)
$$

The frequency $f_{\mathrm{B}}$ calculated by Bohr must add a $1 / 2$ factor to be consistent with the empirical Balmer formula ${ }^{[4,5]}$

$$
f(\tau)=\frac{\tau}{2}
$$

After introducing this "half frequency" factor, Bohr can deduce Balmer formula and Rydberg constant from classical mechanics theory. Therefore, Bohr has great confidence in his classical theory. He insisted that quantum mechanics should be a theory as little as possible divorced from classical mechanics ${ }^{[6]}$. According to the missing radiation mechanism in quantum theory, Bohr tried to restore the relationship between radiation and electron motion in quantum theory similar to classical radiation theory. However, Bohr did not succeed in his life. Not only did he not put forward new hypotheses about the mechanism of radiation, but after 1924 he began to avoid the original 
hypotheses ${ }^{[5]}$. He can only use the limiting case where $n \rightarrow \infty, n-1 \rightarrow n$, under high quantum number to weaken the "half frequency" difference, which is the correspondence principle he later $\operatorname{advocated}^{[7]}$.

\section{Lorentz force in hydrogen atom}

By this paper it is found that the "half frequency" problem perplexing Bohr's whole life is due to a fact that Bohr only considers the electrostatic Coulomb force $\left(F_{\mathrm{C}}\right)$ between electron and proton in his theoretical derivation (Fig. 1A) . The electron in the hydrogen atom is moving, and there must be a magnetic field $\boldsymbol{B}$ in the atom. In addition to Coulomb force $F_{\mathrm{C}}$, electrons in atoms are bound to be affected by Lorentz force $F_{\mathrm{L}}$.
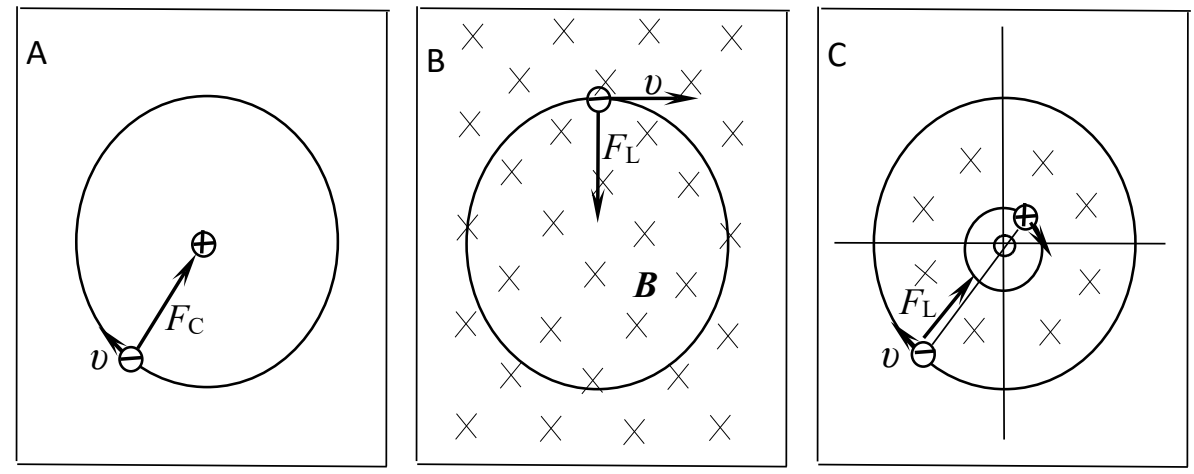

Fig.-1. A) Bohr model, the centripetal force for electronic motion is provided by Coulomb force $F_{\mathrm{C}}$ only. B) Lorentz force $F_{\mathrm{L}}$ which no nuclear charge is required provides centripetal force. C) The Lorentz force $F_{\mathrm{L}}$ produced by magnetic field $\boldsymbol{B}$ of the magnetic moment of the proton orbit can provide centripetal force.

In Fig.-1A, the generation for the Coulomb force does not need the motion of electron, which only produces centrifugal force to balance $F_{\mathrm{C}}$. In Fig.-1B, the generation for the Lorentz force does not need nuclear charge, and the $F_{\mathrm{L}}$ only depends on the magnetic field $\boldsymbol{B}$ and the velocity of electron $\boldsymbol{v}$. Lorentz force can also provide centripetal force for electron in circular motion. The Coulomb force and the Lorentz force are two independent forces, and both they should exist in the hydrogen atom.

If the electron charge is $q$, the electron velocity is $v$, the electric field is $\boldsymbol{E}$ and the magnetic field is $\boldsymbol{B}$, then the force $\boldsymbol{F}$ on the electron can be given by the following Lorentz equation

$$
\boldsymbol{F}=\boldsymbol{F}_{\mathrm{C}}+\boldsymbol{F}_{\mathrm{L}}=q \boldsymbol{E}+q(\boldsymbol{v} \times \boldsymbol{B})
$$

In formula (14)

$$
F_{\mathrm{C}}=q E, \quad F_{\mathrm{L}}=q(v \times B)
$$


Bohr did not consider $\boldsymbol{F}_{\mathrm{L}}$, only $\boldsymbol{F}_{\mathrm{C}}$

$$
F_{\mathrm{C}}=q E=k \frac{e^{2}}{r_{B}^{2}}
$$

where $k$ is the Coulomb constant. The magnetic field $\boldsymbol{B}$ felt by electrons in hydrogen atom mainly comes from the orbital and spin magnetic moments of protons. The magnitude and direction of proton spin moment are relatively clear and simple, and the magnetic field direction generated by proton orbital moment can be analyzed with the help of Fig.-1C. If protons and electrons move in a clockwise circle around their common center of mass, the motion of protons forms an annular current. The right-hand rule can determine that the magnetic field generated by protons is perpendicular to the paper plane and inward. According to the left-hand rule, the direction of Lorentz force $\left(F_{\mathrm{L}}\right)$ on the electron points to the center along the radius.

We don't know the values of $\boldsymbol{B}$ in formula (14) and (15). However, we can directly analyze the existence and magnitude of $F_{\mathrm{L}}$ from following three aspects.

First, as shown in Fig.-1B, it is unrealistic to require a uniform magnetic field $\boldsymbol{B}$ in the whole atom. However, it is possible that there is an equal magnetic field $\boldsymbol{B}$ along the orbit of the electron, which is synchronous with the motion of the electron.

Second, we can learn about electrons by photons. In isotropic space, the electromagnetic energy of photon is ${ }^{[8]}$

$$
\vec{E} \cdot \frac{\partial \vec{D}}{\partial t}+\vec{H} \cdot \frac{\partial \vec{B}}{\partial t}=\left(\frac{1}{2} \varepsilon E^{2}+\frac{1}{2} \mu H^{2}\right)
$$

where $\vec{E}, \vec{H}, \vec{D}, \vec{B}, \varepsilon$ and $\mu$ are electric field strength, magnetic field strength, electric energy density, magnetic flux density, dielectric constant and permeability, respectively. The electric field energy $\frac{1}{2} \varepsilon E^{2}$ and magnetic field energy $\frac{1}{2} \mu H^{2}$ for a photon are not only symmetrical in form, but also equal in energy. Therefore, we speculate that the $F_{\mathrm{C}}$ and $F_{\mathrm{L}}$ felt by the electron in stable orbit of hydrogen atoms should also be approximately equal.

Third, theoretically, $\boldsymbol{F}_{\mathrm{C}}$ and $\boldsymbol{F}_{\mathrm{L}}$ can independently provide centripetal force for the circular motion of electron respectively. Namely

$$
F_{\mathrm{C}}=k \frac{e^{2}}{r^{2}}=m \frac{v^{2}}{r}
$$




$$
F_{L}=e(v \times B)=m \frac{v^{2}}{r}
$$

When $\boldsymbol{F}_{\mathrm{C}}$ and $\boldsymbol{F}_{\mathrm{L}}$ coexist in a hydrogen atom and reach equilibrium with the circular motion of electron, they must exist the following relationships

$$
\left\{\begin{array}{l}
F_{\mathrm{C}}=F_{L} \\
F_{\mathrm{C}}+F_{L}=m \frac{v^{2}}{r}
\end{array}\right.
$$

By formula (20) and (21) we get

$$
2 F_{\mathrm{C}}=2 F_{\mathrm{L}}=\frac{2 k e^{2}}{r^{2}}=2 e(v \times B)=m \frac{v^{2}}{r}
$$

By equation (22), we can get the orbit radius $r$ for the ground state hydrogen atom and the magnetic field intensity $\boldsymbol{B}$ felt by the electron in the electrodynamic model.

$$
\begin{aligned}
& r=\frac{2 k e^{2}}{m v^{2}} \\
& =\frac{2 \times 8.988 \times 10^{9} \times\left(1.602 \times 10^{-19}\right)^{2}}{9.110 \times 10^{-31}\left(2.188 \times 10^{6}\right)^{2}}=1.0583 \times 10^{-10}(\mathrm{~m}) \\
& B=\frac{m v}{2 e r} \\
& =\frac{9.110 \times 10^{-31} \times 2.188 \times 10^{6}}{2 \times 1.602 \times 10^{-19} \times 1.0583 \times 10^{-10}}=5.878 \times 10^{4}(\mathrm{~T})
\end{aligned}
$$

Because the electron moves in a circular orbit in the ground state, we can calculate the rotation frequency of the electron $(f)$ by the following formula

$$
f=\frac{v}{2 \pi r}=\frac{2.188 \times 10^{6}}{2 \pi \times 1.0583 \times 10^{-10}}=3.290 \times 10^{15}\left(\mathrm{~s}^{-1}\right)
$$

Comparing equation (23) with equation (6) and equation (25) with equation (4), both $r$ and $f$ calculated here are completely equal to the both Rydberg radius $r_{\mathrm{R}}$ and Rydberg frequency $f_{\mathrm{R}}$ calculated according to the experimental data above section 2 .

In this derivation, we use the formula (20), that is, $\boldsymbol{F}_{\mathrm{C}}=\boldsymbol{F}_{\mathrm{L}}$ in the ground state hydrogen atom. When the hydrogen atom is in the external electric or magnetic field, the forces felt by the electron, $\boldsymbol{F}_{\mathrm{C}} \neq \boldsymbol{F}_{\mathrm{L}}$, which will split the energy levels and the spectral lines. The splitting in the applied electric field is 
called Stark effect, and the splitting in the applied magnetic field is called Zeeman effect ${ }^{[9]}$.

Here, we have completely solved and eliminated the "half frequency" problem encountered in Bohr's theory. On the other hand, it also proves that Bohr's idea of using classical mechanics to explain radiation mechanism is correct.

\section{Force analysis for electron in photon field}

Now we can boldly follow Bohr's original idea to discuss both the atomic radiation mechanism and details by the classical mechanics and the standing wave theory in quantum mechanics.

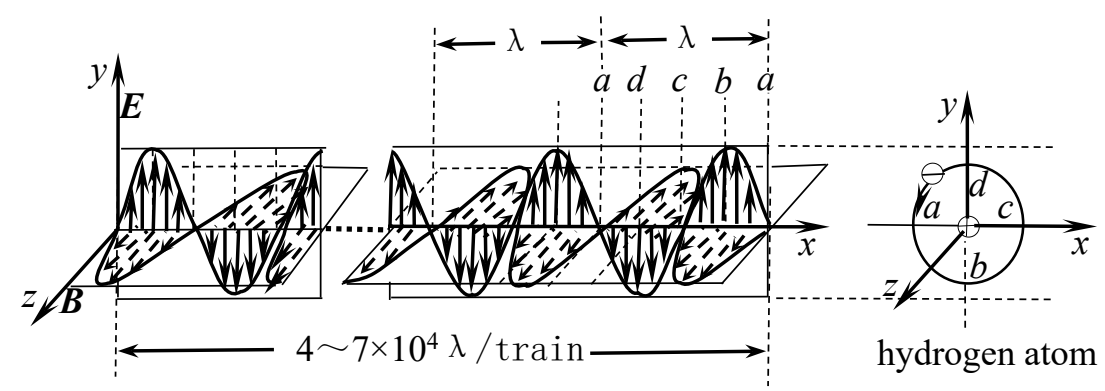

Fig.-2. On the left, only a few wavelengths in the head and tail of a photon train are represented by the Poynting vectors. The $\boldsymbol{E}$ vector in the photon is in the same plane with the orbit and the $\boldsymbol{B}$ vector is perpendicular to this plane. On the right is the hydrogen atom before absorbing the photon.

A photon can be regarded as a long wave train represented by Poynting vector ${ }^{[10]}$. According to the duration of the atomic emission $\tau_{0} \approx 10^{-10}$ seconds ${ }^{[11,12]}$, we can estimate that the length of a photon wave train of visible light is about $4 \sim 7 \times 10^{4}$ wavelengths. As shown in Fig.-2, for photon the electric vector $\boldsymbol{E}$ and magnetic vector $\boldsymbol{B}$ are perpendicular to the direction of light motion respectively. The spatial distribution of the three vectors determines that photons have chirality, so they exhibit a property of polarization when propagating in chiral media (such as glucose solution , sucrose solution, etc.). Figure 2 shows that a photon will be absorbed by a ground state hydrogen atom, and the electron orbits counterclockwise. In the absorption collision, the four phases $a, b, c$ and $d$ in the photon period are roughly synchronized with the four phases $a, b, c$ and $d$ in the orbital period of the ground state hydrogen atom. 


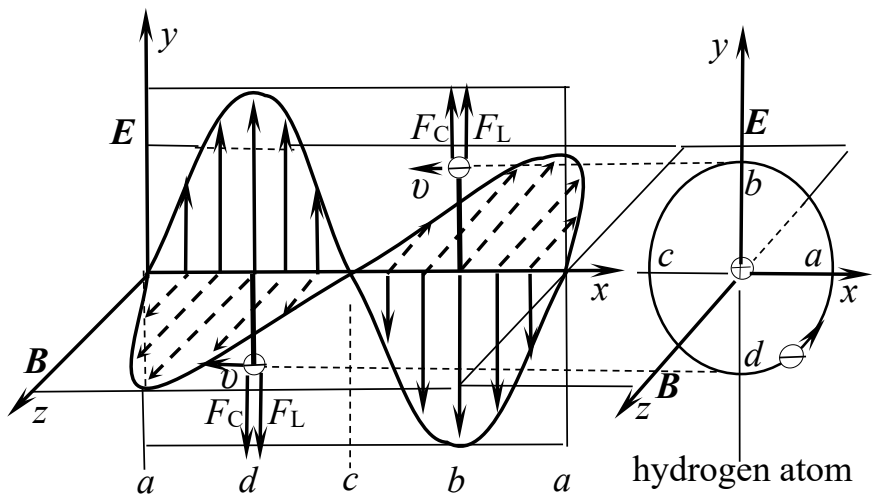

Fig.3. In a process of absorption collision, the photon moves to the right, which is equivalent to the electron moving to the left at the same speed into the photon field. The direction of Coulomb force is the same with of Lorentz force, both the two forces are to move the electron far away from the atomic nucleus along directions of $+y$ and $-y$, respectively, and the period of revolution enlarges with the increase of radius.

Figure-3 shows the interaction of a wave field of a photon wave train propagating in the $x$ direction with electron in the hydrogen atom. The photon movement to the right is equivalent to the electron moving to the left at approximately the same speed (compared with the speed of light, the velocity of the electron in orbit can be ignored). For an isolated hydrogen atom, the electron is mainly affected by three fields: nuclear field $\boldsymbol{E}_{\mathrm{N}}$, photon $\boldsymbol{B}$ field and photon $\boldsymbol{E}$ field.

\section{1) Nuclear electric field $E_{N}$}

When an electron moves in a circle around the nucleus, the nuclear electric field only provides centripetal force, and Coulomb force is perpendicular to the velocity of electron motion, and does not change the electron velocity.

When an electron moves elliptically around the nucleus, the Coulomb force produces a component in the direction of the electron velocity. When the electron goes far, it slows down the electron, and it accelerates when it approaches. The nuclear electric field is a conservative potential field, which does not change the average velocity of electrons.

\section{2) Photon $B$ field}

In Figure 3, when the electron moves to the left and reaches point $a$, the field strength of photon $\boldsymbol{B}$ field is zero, and the electron is not forced. When the electron moves from $a$ to $b$, the photon $\boldsymbol{B}$ field increases gradually, and the direction is perpendicular to the paper plane and inward. The direction 
of the Lorentz force on the electron is always perpendicular to the direction of motion, that is, along the radius outward, and the component in the $y$ direction gradually increases. When the electron reaches the $b$ point, the Lorentz force of the photon field is the largest, and its direction is outward along $+y$. The electron is pulled away from the nucleus and the orbital radius reaches maximum first.

From point $b$ to point $c$, the photon $\boldsymbol{B}$ field gradually weakens, and decreases to zero when reaches point $c$. After past the point $c$, the sign reversal of the photon $\boldsymbol{B}$ field. From point $c$ to $d$, the sign of Lorentz force component felt by the electron in the $y$ direction also reverses, pointing to $-y$ direction, still pulling the electron away from the nucleus. The Lorentz force and orbit radius reach the maximum again at point $d$. From $d$ to $a$, the Lorentz force and the orbital radius decrease gradually, and the orbital radius returns to the value at the beginning of the period when it reaches point $a$ again. The interaction of the $\boldsymbol{B}$ field of one of waves in the photon wave train with one period of the electron only makes the orbit elongate a little along the $+y$ and $-y$ directions.

\section{3) Photon $E$ field}

The Coulomb force exerted by photon $\boldsymbol{E}$ field on electrons can not only increase the radius of the orbit along the direction of $+\mathrm{y}$ and-y, but also change the velocity of electrons. In Fig.-4A and -4B, the "-" and "+" represent the negative and positive photon $\boldsymbol{E}$ fields respectively. The photon field moves to the right ( $+x$ direction) at the speed of light, which is equivalent to the electron moving to the left ( $-x$ direction) at the same speed. Compared with the speed of light, the speed of electrons around the nucleus can be ignored. In Fig.-4A, in the first half circle $(a \rightarrow b \rightarrow c)$, the electron feels negative photon $\boldsymbol{E}$ field. It is equivalent to shielding a part of the nuclear charge, which produces

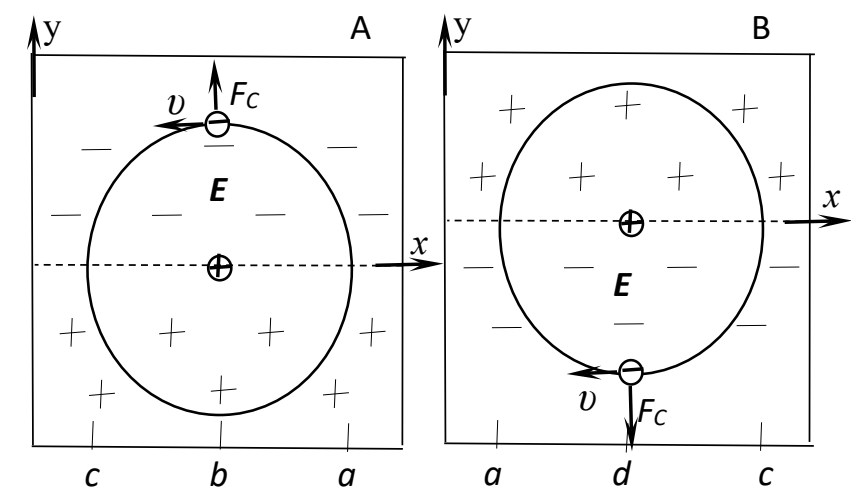

Fig.4. A) The direction of the photon $\boldsymbol{E}$ field force felt by the electron at $b$ point. B) The direction of the photon $\boldsymbol{E}$ field force felt by the electron at $d$ point. 
repulsive force and motion resistance to the electron, which increases the radius of the orbit in the $+y$ direction and decreases the speed of the electron movement.

In Fig.-4B, the electron passes through point $c$, and the symbol of photon $\boldsymbol{E}$ field reverses. In the second half circle $(c \rightarrow d \rightarrow a)$, the electron still feels negative photon $\boldsymbol{E}$ field, and the orbital radius increases along the $-y$ direction and the electron motion speed decreases. When it returns to point $a$ again, the photon wave train and the orbital rotation of the electron will enter the next period.

Under the influence of the three fields $\left(\boldsymbol{E}_{\mathrm{N}}, \boldsymbol{B}\right.$ and $\left.\boldsymbol{E}\right)$, when a period is completed, the radius of the orbit along the $x$ direction is not changed, but the radius of the orbit along the $y$ direction is obviously enlarged. However, the average radius only increased a little per period. For each additional period, the electron velocities at points $a$ and c remain almost unchanged, while the electron velocities at points $b$ and $d$ decrease a little, but the average velocity decreases even less.

\section{The orbital change of electron in photon field}

In classical physics, forces and motions can be synthesized or decomposed by vector law. A circular motion and a directional motion can synthesize the elliptical motion. Similarly, an ellipse motion can be divided into a circular motion and a directional motion. Figure 5 is a schematic diagram of orbit change of the earth satellite. In Fig.-5A, if only a directional force $\boldsymbol{F}$ is applied to the satellite in the $a$-point of

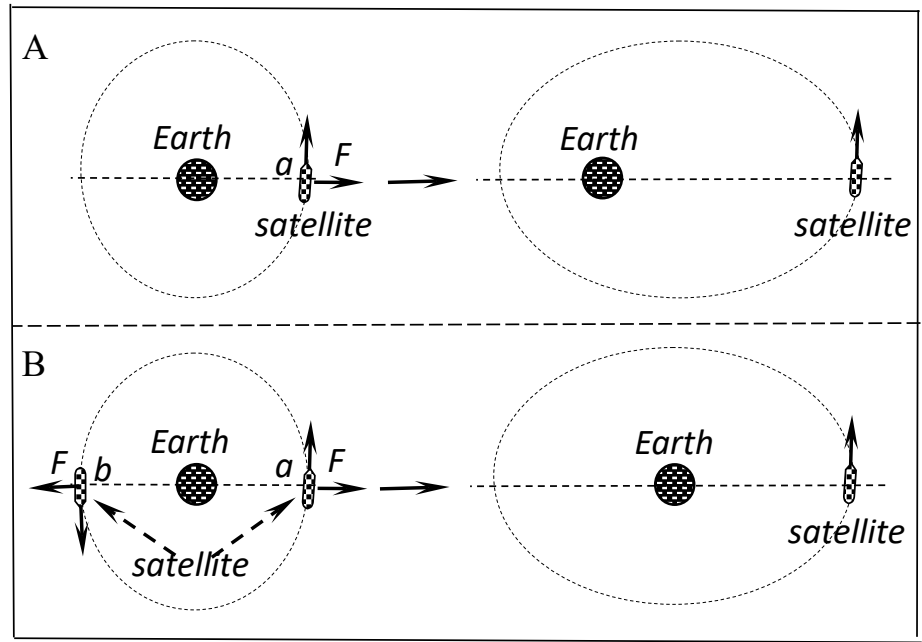

Fig.-5. Schematic diagram of earth satellite orbit change. A) shows that if we change orbit only at point $a$, the earth will be in a focal point of the elliptical orbit; $B$ ) shows that if change orbit with the same force at point $a$ and $b$, respectively, the earth will be at the midpoint of the elliptical orbit. The effect of photons on electrons is similar to the $B$ ).

the circular orbit to change its orbit, the circular orbit will be changed into elliptical orbit, and the earth 
will be in one of two focuses of the ellipse. In Fig.-5B, if two forces with same size are applied at point $a$ and $b$ of the circular orbit respectively, it will also become elliptical orbit, but the earth will be at the midpoint of the ellipse. The effect of photon field on electron is similar to that in Fig.-5B. That is to say, whether in circular orbit or elliptical orbit, the nucleus is always at the center of the orbit, not at a focal point of the elliptical orbit.

The effect of a photon on electron is a kind of lateral directional motion superimposed on the original orbit on the same plane. As shown in Fig. 6, the photon wave train moves to the right at the speed of light along the $x$ direction, which is equal to the electron moving to the left at the same speed. In the course of interaction, the diameter of the orbit along the $x$ direction remains unchanged. Therefore, the time period of the photon wave train can repeat on the orbit along the $x$ direction, which makes the photon and electron basically keep synchronous in the main interaction period.

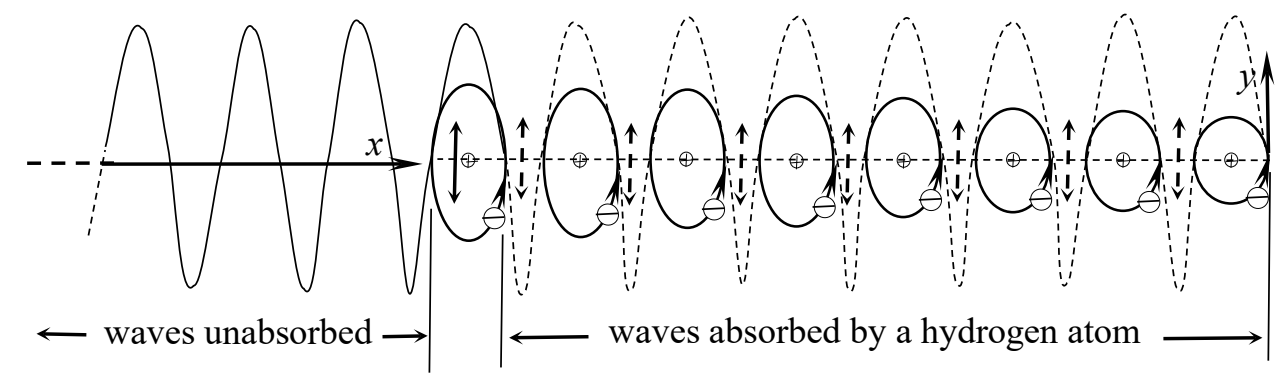

Fig. 6. During electron transition, the orbital width of the hydrogen atom is changeless on the direction of photon movement, therefore, it is in sync with photon.

Although one wavelength of a photon wave train can only lengthen the electron's orbit a little along the $y$ direction, when the photon wave train with tens of thousands or more wavelengths is absorbed by an electron, the circular orbit of the electron will be pulled into an ellipse. The force $\boldsymbol{F}_{y}$ exerted by photon field on electron along the y-axis consists of photon $\boldsymbol{B}$ field and photon $\boldsymbol{E}$ field.

$$
F_{y}=F_{\mathrm{L} y}+F_{\mathrm{C} y},
$$

The magnitude of $F_{y}$ is mainly affected by the phase difference $(\Delta \varphi)$ between electron and photon. The $\Delta \varphi$ can be calculated according to the following formula

$$
\Delta \varphi=\frac{\pi\left(f_{n 1}-v\right)}{f_{n 1}}
$$

If $\Delta \varphi$ is a quarter of a cycle, $(\pi / 2), F_{y}=0$. If a photon is fully synchronized with the electron, i.e, the phase difference is $0, F_{y}=F_{\max }$. 


$$
F_{y}=F_{\max } \cos \frac{\pi\left(f_{n 1}-v\right)}{f_{n 1}}
$$

For example, for the Lyman series,

$$
\begin{array}{r}
n_{1}=1, f_{\mathrm{n} 1}=f, \text { and } v_{\alpha}=\frac{3}{4} f, \quad v_{\beta}=\frac{8}{9} f, \quad v_{\gamma}=\frac{15}{16} f, \quad v_{\delta}=\frac{24}{25} f, \\
F_{\mathrm{y} \alpha}=F_{\max } \cos \frac{\pi\left(f-\frac{3}{4} f\right)}{f}=F_{\max } \cos \frac{\pi}{4}
\end{array}
$$

and $F_{\mathrm{y} \beta}=F_{\max } \cos \frac{\pi}{9}, F_{\mathrm{yy}}=F_{\max } \cos \frac{\pi}{16}, F_{\mathrm{y} \delta}=F_{\max } \cos \frac{\pi}{25}, \ldots \ldots$

当 $n_{2} \rightarrow \infty, v \rightarrow f$,

$$
F_{\mathrm{y} \infty \mathrm{o}}=F_{\max } \cos \frac{\pi(f-f)}{f}=F_{\max }
$$

For the Balmer series. $n_{1}=2, f_{\mathrm{n} 1}=f / 4, \quad$ and $v_{\mathrm{H} \alpha}=\frac{5}{36} f, \quad v_{\mathrm{H} \beta}=\frac{3}{16} f, \quad v_{\mathrm{H} \gamma}=\frac{21}{100} f, \quad v_{\mathrm{H} \delta}=\frac{2}{9} f, \ldots \ldots$ $F_{\mathrm{y} \alpha}=F_{\mathrm{B} \max } \cos \frac{\pi}{9}, F_{\mathrm{y} \beta}=F_{\mathrm{B} \max } \cos \frac{\pi}{16}, F_{\mathrm{y} \beta}=F_{\mathrm{B} \max } \cos \frac{\pi}{25}, F_{\mathrm{y} \beta}=F_{\mathrm{B} \max } \cos \frac{\pi}{36}, \ldots \ldots$

当 $n_{2} \rightarrow \infty, v \rightarrow f / 4$,

$$
F_{\mathrm{y} x}=F_{\mathrm{B} \max } \cos \frac{\pi\left(\frac{f}{4}-\frac{f}{4}\right)}{\frac{f}{4}}=F_{\mathrm{B} \max }
$$

$F_{y}$ is a transverse force perpendicular to the direction of photon motion. According to Newton's second law, it can make electrons accelerate in the direction of force.

$$
F_{\mathrm{y}}=m a_{\mathrm{y}}
$$

If their common period is $T$, then the long half axis of orbit per period will increase

$$
\begin{gathered}
\Delta r_{y}=\frac{1}{2} a_{\mathrm{y}} T^{2} \\
\because T=1 / v, \therefore \Delta r_{y}=\frac{a_{y}}{2 v^{2}}
\end{gathered}
$$

A photon wave train has $\tau_{0} v$ of waves, when it is completely absorbed by an electron, the long half axis of the orbit increases

$$
\Delta R_{y}=\frac{\tau_{0} a_{y}}{2 v}
$$


In the following section 8 , we will prove the $\Delta$ Ry is quantized, and $r_{n}=(2 n-1) r$ along the $y$ axis.

Therefor, we have

$$
\Delta R_{y}=\frac{\tau_{0} a_{y}}{2 v}=2\left(n_{2}-n_{1}\right) r
$$

For example, for $\mathrm{H}_{\alpha}$ line in the Balmer serises, $n_{1}=2, n_{2}=3, v=4.569 \times 10^{14} \mathrm{~s}^{-1}$, by formula (36)

$$
\begin{aligned}
& a_{y}=\frac{4\left(n_{2}-n_{1}\right) v r}{\tau_{0}} \\
& =\frac{4(3-2) \times 4.569 \times 10^{14} \times 1.059 \times 10^{-10}}{10^{-10}}=1.935 \times 10^{15}\left(\mathrm{~ms}^{-2}\right)
\end{aligned}
$$

By formula (33) we get

$$
\begin{aligned}
& \Delta r_{y}=\frac{1}{2} a_{\mathrm{y}} T^{2} \\
& =\frac{1.935 \times 10^{15}}{2\left(4.569 \times 10^{14}\right)^{2}}=4.634 \times 10^{-15}(\mathrm{~m})
\end{aligned}
$$

The parameters of electron orbit change related to four main spectral lines in Lyman series and Balmer series respectively are listed in Table-1. Of course, the accuracy of the orbital parameters related to each spectral line in the table also depends on the accurate determination of the wave train length (or $\left.\tau_{0}\right)$ in the future. This calculation and analysis only show that the motion state of micro particles can also be calculated and treated by classical mechanics.

Table 1. Orbital parameters of electrons related to the main spectral lines in hydrogen atom.

\begin{tabular}{|lccccccrrrrr|}
\hline $\begin{array}{l}\text { Spectral } \\
\text { series }\end{array}$ & Line & $n_{1}$ & $n_{2}$ & $2\left(n_{2}-n_{1}\right)$ & $\lambda(\mathrm{nm})$ & $\begin{array}{c}v=c / \lambda \\
a_{y}=4 v r\left(n_{2}-n_{1}\right) / \tau_{0}\end{array}$ & $\Delta r=0.5 a_{y} T^{2}$ & $N=\tau_{0} v$ & $\Delta R=\tau_{0} v \Delta r$ \\
\hline & $\alpha$ & 1 & 2 & 2 & 121.502 & 2.4674 & 10.4419 & 0.8576 & 2.4674 & 2.1160 \\
Lyman & $\beta$ & 1 & 3 & 4 & 102.521 & 2.9242 & 24.7503 & 1.4472 & 2.9242 & 4.2320 \\
series & $\gamma$ & 1 & 4 & 6 & 97.202 & 3.0842 & 39.1569 & 2.0582 & 3.0842 & 6.3480 \\
& $\delta$ & 1 & 5 & 8 & 94.924 & 3.1582 & 53.4622 & 2.6800 & 3.1582 & 8.4640 \\
\hline \multirow{2}{*}{ Balmer } & $\mathrm{H} \alpha$ & 2 & 3 & 2 & 656.112 & 0.45692 & 1.9337 & 4.6310 & 0.4569 & 2.1160 \\
series & $\mathrm{H} \beta$ & 2 & 4 & 4 & 486.009 & 0.61684 & 5.2209 & 6.8608 & 0.6168 & 4.2320 \\
& $\mathrm{H} \gamma$ & 2 & 5 & 6 & 433.937 & 0.69086 & 8.7712 & 9.1885 & 0.6909 & 6.3480 \\
& $\mathrm{H} \delta$ & 2 & 6 & 8 & 410.070 & 0.73107 & 12.3756 & 11.5775 & 0.7311 & 8.4640 \\
\hline
\end{tabular}




\section{In the process of electron transition the change of orbital angular momentum and the} physical meaning of Planck constant $h$

When the electron moves in the steady orbit, the angular momentum is conserved, the rotation frequency is constant, and it is in the standing wave state, so it neither radiates nor absorbs photons. However, the angular momentum is not conserved in the process of photon-electron interaction. When both the photon wave train and the rotation of electron common complete one cycle ( $T$ or 2 $\pi$ ), the orbit is elongated a little along the y-axis, and during the period $T$, the angular momentum changes by a Planck energy quantity $(\mathcal{H})$. That is,

$$
\int_{0}^{2 \pi} \frac{m v}{T} d r=\frac{2 \pi m v r}{T}=\mathcal{H}
$$

When the electron transitions from level $n_{1}$ to $n_{2}$ during $\tau_{0}$, the period number of interaction for the electron with the photon wave train is

$$
v \tau_{0}=\left(f_{n 1}-f_{n 2}\right) \tau_{0}
$$

During $\tau_{0}$ the change of the total orbital angular momentum after the electron transition leads to the change of orbital energy level

$$
\int_{0}^{2 \pi v \tau_{0}} \frac{m v}{T} d r=v \tau_{0} \frac{2 \pi m v r}{T}=\left(f_{n 1}-f_{n 2}\right) \tau_{0} \mathcal{H}
$$

The change of angular momentum per unit time is also the change of energy, $\Delta E_{n 1 \rightarrow n 2}$, and the expression (41) is also the radiation energy in the process of electron transition

$$
\Delta E_{n 1 \rightarrow n 2}=\left(f_{n 1}-f_{n 2}\right) \tau_{0} \mathcal{H}=\tau_{0} \mathcal{H} v
$$

In the current textbooks, the energy expression of photons related to electron transition is

$$
\Delta E_{n 1 \rightarrow n 2}=h v
$$

Comparing the formula (43) with (42), we get

$$
\begin{gathered}
\tau_{0} \mathcal{H} v=h v \\
\mathcal{H}=h / \tau_{0} \\
\approx \frac{6.626 \times 10^{-34} \mathrm{~J} \cdot \mathrm{s}}{10^{-10} \mathrm{~s}}=6.626 \times 10^{-24} \mathrm{~J}
\end{gathered}
$$

It can be seen that $\mathcal{H}$ is about $10^{10}$ times of Planck constant $h$ numerically. The unit of the $\mathcal{H}$ is Joule $(\mathrm{J})$, which has nothing to do with time, and the $\mathcal{H}$ is the minimum energy absorbed or released by an electron to rotate around the orbit one time during the transition. The unit of the $h$ is 
Joule $\cdot \operatorname{second}(\mathrm{J} \cdot \mathrm{S})$, but the physical meaning of the $h$ is not clear. In the formula (42), the time contained in the term $\tau_{0} \mathcal{H}$ is $10^{-10}$ seconds, and the time contained in the $h$ in formula (43) is second (S). $\tau_{0}$ is much less than 1 second.

To illustrate the influence of this time unit selection, take an example. A school has eight classes in eight hours during the day time, including one class every hour during recess. If calculated by day, there is no doubt that there are eight classes per day. However, the statement of 8 classes per day does not consider the details of classes, because on average, there are only 1 / 3 classes per hour (including recess) according to 24 hours per day. Of course, this is not in line with the actual situation. The Planck constant $h$ is similar to the case of the $1 / 3$ classes per hour, and the photon frequency is similar to the case of eight classes per day.

Because the Planck constant $h$ is determined from the experiment, and during $\tau_{0}$ the events occurred are allocated to each second artificially, there is no problem in data measurement, but the details are not in line with the actual situation. Therefore, the physical meaning of Planck constant $h$ is not clear so far. After understanding the $\mathcal{H}$, here, the physical meaning of Planck constant $h$ is clear at a glance.

\section{By standing wave principle to quantize the energy levels of hydrogen atom}

According to the standing wave principle of quantum mechanics, waves from different sources (or directions) will be superimposed on each other and converge at the boundary of the standing wave potential box. The hydrogen atom can be regarded as a three-dimensional standing wave potential box. In the ground state, the diameter can be regarded as the length of the potential box. As shown in figure-7, when the hydrogen atom absorbs photon, the width of the potential box in the direction of photon motion ( $x$-axis) remains unchanged. The photon is a transverse wave and only elongates the potential box along the y-axis. Standing wave condition requires that the stretching of potential box must be quantized. If the length of the potential box is $2 r$, the radius along the $+y$ or $-y$ direction can only be increased by an integral multiple of $2 r$. In other words, the short half axis of the new orbit remains unchanged, but the long half axis can only be $r, 3 r, 5 r, 7 r, 9 r, \ldots \ldots,(2 \mathrm{n}-1) r$.

The average radius of the $n$th stable orbit is

$$
\bar{r}_{n}=\frac{(2 n-1) r+r}{2}=n r
$$

The orbital angular momentum (mvr) of the electron at the steady state level conserves, at points $a$ 
and $c, r_{n}=r, v_{n}=v$; at points $b$ and $d, \because r_{n}=(2 n-1) r, \therefore v_{n}=v /(2 n-1)$. The average orbital velocity of the electron of the $n$th energy level

$$
\bar{v}_{n}=\frac{v}{n}
$$

For circular orbit of the ground state hydrogen atom, there is

$$
f=v /(2 \pi \mathrm{r})
$$

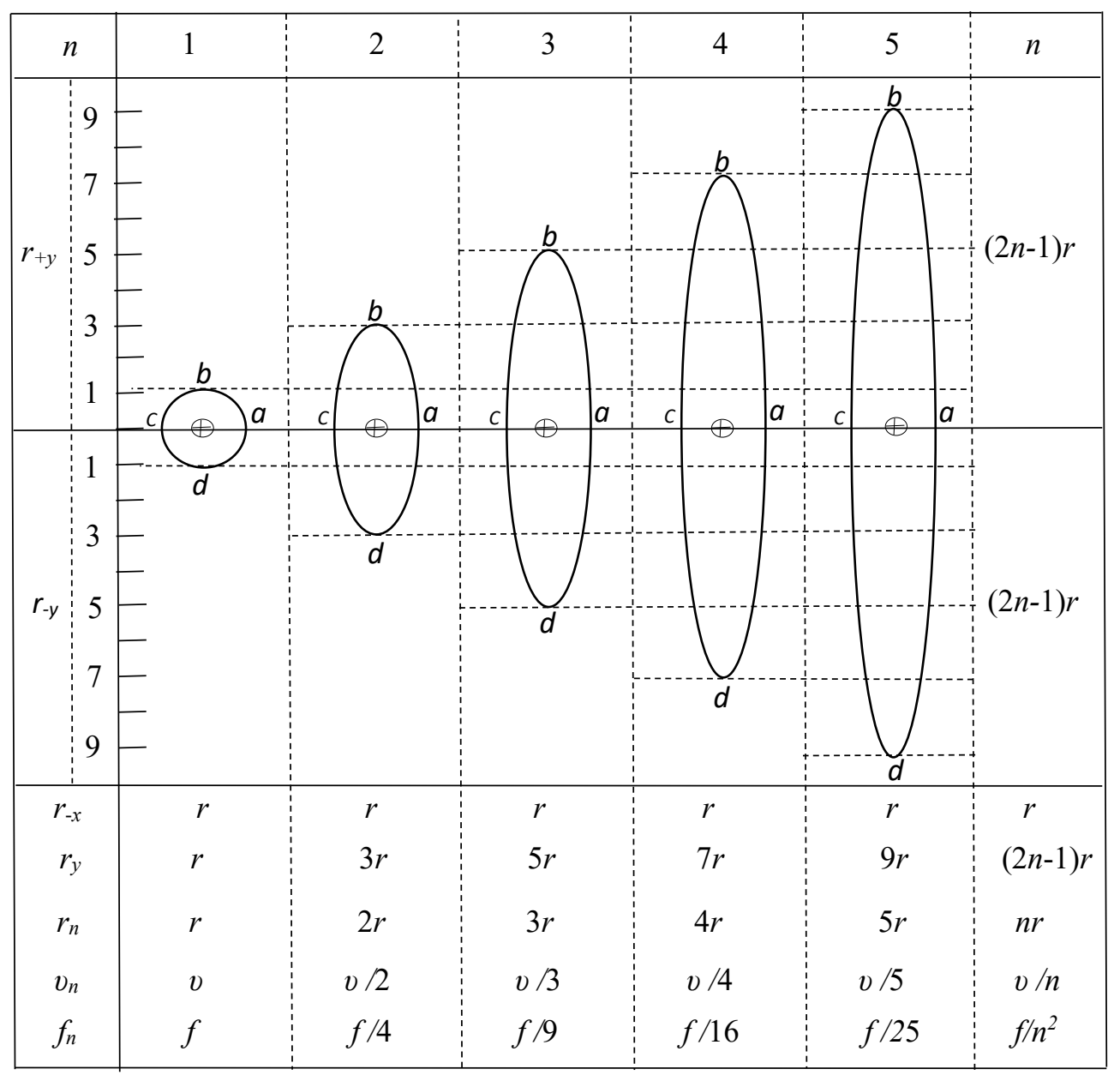

Fig.-7. The orbital parameters of different energy level of hydrogen atom. Short radius, long radius, average radius, average velocity and orbit frequency are expressed as $r_{x}, r_{y}$, $r_{n}, v_{n}$, and $f_{n}$, respectively. The length of each of the potential boxes is an integral multiple of $2 r$. Using the wave equation and the current quantum mechanics theory, only the bottom line data (orbital energy or frequency expression) are obtained accurately.

When the average radius and average velocity are used to an elliptical orbit, the orbit frequency can also be calculated according to the circular motion.

$$
f_{n}=\frac{\bar{v}_{n}}{2 \pi \bar{r}_{n}}=\frac{f}{n^{2}}
$$


The ratio of the $f$ to the speed of light $(c)$ is the Rydberg constant. From the energy point of view, when the electron transitions from the high energy level $n_{2}$ to the low energy level $n_{1}$, it will radiate photon. The energy of the photon is

$$
E=h v=h\left(f_{n 1}-f_{n 2}\right)=h f\left(\frac{1}{n_{1}^{2}}-\frac{1}{n_{2}^{2}}\right)
$$

The photon frequency in equation (50) is just equal to the difference between the orbital frequencies of the two energy levels

$$
v=f_{n 1}-f_{n 2}
$$

Equations (50), (51) are consistent with equations (3).

\section{Width of spectral line}

The linewidth of spectral line $(\Delta \lambda)$ refers to the difference between two adjacent wavelengths in a photon wave train. There are the following relations between the linewidth $(\Delta \lambda)$ and the length of photon wave train $(\mathrm{L})$ and wavelength $(\lambda)^{[10,13]}$

$$
\frac{L}{\lambda}=\frac{\lambda}{\Delta \lambda}=v \tau_{0}
$$

We denote the first wavelength of the head of a wave train as $\Lambda_{1}$, the frequency as $f_{1}$; and denote the last wavelength in the tail as $\Lambda_{2}$ and the frequency as $f_{2}$. Then, equation (52) shows that the difference between the train head $\Lambda_{1}$ and the train tail $\Lambda_{2}$ is only one wavelength. Then, there must be

$$
f_{1}=2 f_{2} \quad \text { or } 2 f_{1}=f_{2}
$$

Let's set $f_{1}=v+x, f_{2}=v-x$, and $f_{1}=2 f_{2}$, by solving the following simple algebraic equation,

$$
v+x=2(v-x)
$$

we obtain

$$
\begin{gathered}
x=\frac{1}{3} v \\
f_{1}=\left(1+\frac{1}{3}\right) v=\frac{4}{3}\left(\frac{f}{n_{1}^{2}}-\frac{f}{n_{2}^{2}}\right)=\frac{4 f}{3}\left(\frac{1}{n_{1}^{2}}-\frac{1}{n_{2}^{2}}\right) \sqsubset \quad \Lambda_{1}=\frac{c}{f_{1}}=\frac{3 c}{4 f} \cdot \frac{n_{1}^{2} \cdot n_{2}^{2}}{n_{2}^{2}-n_{1}^{2}} \\
f_{2}=\left(1-\frac{1}{3}\right) v=\frac{2}{3}\left(\frac{f}{n_{1}^{2}}-\frac{f}{n_{2}^{2}}\right)=\frac{2 f}{3}\left(\frac{1}{n_{1}^{2}}-\frac{1}{n_{2}^{2}}\right) \sqsubset \quad \Lambda_{2}=\frac{c}{f_{2}}=\frac{3 c}{2 f} \cdot \frac{n_{1}^{2} \cdot n_{2}^{2}}{n_{2}^{2}-n_{1}^{2}}
\end{gathered}
$$

For example, for $\mathrm{H}_{\alpha}$ line $n_{1}=2, n_{2}=3, v=4.569 \times 10^{14} \mathrm{~s}^{-1}, \lambda=656.1 \mathrm{~nm}$,

$$
\begin{aligned}
& f_{1}=\frac{4 f}{3}\left(\frac{1}{n_{1}^{2}}-\frac{1}{n_{2}^{2}}\right) \\
& =\frac{4 \times 3.2899 \times 10^{15}}{3} \cdot \frac{5}{36}=6.092 \times 10^{14}\left(\mathrm{~s}^{-1}\right)
\end{aligned}
$$




$$
\begin{aligned}
& \Lambda_{1}=\frac{3 c}{4 f} \cdot \frac{n_{1}^{2} \cdot n_{2}^{2}}{n_{2}^{2}-n_{1}^{2}} \\
& =\frac{3 \times 2.998 \times 10^{8}}{4 \times 3.2899 \times 10^{15}} \cdot \frac{36}{5}=4.921 \times 10^{-7}(\mathrm{~m}) \\
& f_{2}=\frac{2 f}{3}\left(\frac{1}{n_{1}^{2}}-\frac{1}{n_{2}^{2}}\right) \\
& =\frac{2 \times 3.2899 \times 10^{15}}{3} \cdot \frac{5}{36}=3.046 \times 10^{14}\left(\mathrm{~s}^{-1}\right) \\
& \Lambda_{2}=\frac{3 c}{2 f} \cdot \frac{n_{1}^{2} \cdot n_{2}^{2}}{n_{2}^{2}-n_{1}^{2}} \\
& =\frac{3 \times 2.998 \times 10^{8}}{2 \times 3.2899 \times 10^{15}} \cdot \frac{36}{5}=9.842 \times 10^{-7}(\mathrm{~m})
\end{aligned}
$$

It can be seen that the frequency of the photon $v$ is the average of $f_{1}$ and $f_{2}$, but the wavelength $\lambda$ is not the average of $\Lambda_{1}$ and $\Lambda_{2}$, and $2 \Lambda_{1}=\Lambda_{2}$ or $\Lambda_{1}=2 \Lambda_{2}$. This is determined by the quantization condition of standing wave. Therefore, for a laser of the same wavelength, it is known from equation (52) that there will be a very small linewidth $\Delta \lambda$, and especially big $\lambda / \Delta \lambda$. That is to say, it has a very large wave train length $L$ and a very large action time $\tau_{0}$ 。

$$
\Delta \lambda=\frac{\lambda}{v \tau_{0}}
$$

The $\lambda / \Delta \lambda$ represents the wave number of the whole wave train and its reciprocal $(\Delta \lambda / \lambda)$ determines the accuracy of photon wavelength measurement. For example, in the $1850 \mathrm{~s}$, the Swedish spectrologist Angstrom measured the wavelengths of four visible spectra of hydrogen atoms, which reached 5 6 significant digits ${ }^{[1]}$. It shows that the wave train length of these spectral lines is at least $10^{5}$ waves. The narrower the line width is, the longer the wave train is, and the higher the accuracy of the measured wavelength is, because there is always about one wavelength difference between the $\Lambda_{1}$ and $\Lambda_{2}$.

\section{Conclusion and perspectives}

Hydrogen atom is the simplest atomic system and the main research object of quantum mechanics. More than a century has passed, the Bohr model and Bohr radius have a wide influence and their incorrect impact is pervasive to University and middle school textbooks, so many 
calculations and conclusions in this paper are naturally inconsistent with the current textbooks. It is generally believed that Bohr's theory uses the knowledge of classical physics and is difficult to be incorporated into quantum mechanics; Quantum mechanics uses too much mathematical language and lacks understanding of physical knowledge. In this paper, we break through this conventional thinking and use as few mathematical language as possible. Based on classical physics knowledge, we establish the direct relationship between radiation and electron motion with the help of standing wave quantization condition. This is a career that Bohr pursued in his life but did not complete. The motion state of micro particles can also be described by the combination of classical mechanics and quantum mechanics. Further research can also prove that the uncertainty principle may be a kind of interference effect of measuring instruments on the motion state of micro particles. The details of photons emitted by hydrogen atom will be discussed later due to paper space limitation.

\section{Acknowledgments}

The author would like to thank Professor Pengxi Li of Weifang Engineering Vocational College for his friendly discussion. I also thank Professor Zhang Jingyi of Guangzhou University and Professor Shuangnan Zhang of Center for Astrophysics of Chinese Academy of Sciences for generously providing the full text of their papers.

\section{Reference}

1. Guo, Y. L. Historical review and new progress of Rydberg constant. Physics. 1, 51-55 (1990) (in Chinese)

2. Guo, Y. L. New progress in the evaluation of basic physical constants. Physics. 3, 129-135 (1989) (in Chinese)

3. Liu, Y. Theoretical value of fine structure constant. Journal of Henan Science and Technology. 10, 172-173 (2014) (in Chinese).

4. Bohr, N. On the Constitution of Atoms and Molecules. Philosophical Magazine Series 6. 26, 1-25.

5. Jähnert, M. The correspondence idea in the early Bohr atom 1913-1915. Annalen der Physik.10-11, A155-A158 (2013)

6. Bokulich, P \& Bokulich, A. Niels Bohr's generalization of classical mechanics. Foundations of Physics 35, 347-371 (2005) 
7. Bokulich, A. Three Puzzles about Bohr's Correspondence Principle, 1-24 (2009). http://philsci-archive.pitt.edu/archive/00004826/01/3Puzzles-BohrCP-Bokulich.pdf

8. Zhang, C. H. Analysis of energy flow in motor with Poynting vector Journal of Taiyuan University of Technology. 2, 21-32 (1980) (in Chinese).

9. Duncan, A. \& Janssen, M. The trouble with orbits: the Stark effect in the old and the new quantum Eprint Arxiv 2014arXiv1404.5341D (2014)

10. Wang, X. E. A single photon model in vacuum. Sparks of scientific wisdom of Chinese Academy of Sciences (2017-12-07). http://idea.cas.cn/viewdoc.action?docid=60667 (in Chinese)

11. Li, W. G., Ye, J. Y. \& Chen, Z. F. Measurement of atomic luminescence duration by interferometry GUANGXI PHYSICS. 4, 29-31 (2009) (in Chinese).

12. Yuan, Q., et al. The experimental measurement of the coherent length for $\mathrm{Na}$ lights and estimation of the light emitting time. Physical Experiment of College. 5, 11-13 (2015) (in Chinese)

13. Mo, W. L. \& Sheng, J. M. Concise college physics, Volume 1, Beijing, Peking University Press, (2005). (in Chinese) 


\section{Supplementary Files}

This is a list of supplementary files associated with this preprint. Click to download.

- WangsTable.docx 